\title{
A comparison of verbal and non-verbal reports of fragmenting visual images'
}

JOHN R. SCHUCK ANO WILLIAM R. LEAHY

IOWA STATE UNIVERSITY

Sixty-eight Ss voluntarily restrained head, eye and blinking movements while viewing a luminous "HB" figure in a darkened room. All Ss were told that voluntary fixation would cause parts of the pattern to disappear; they were to report the parts that remained intact. Half of the $S$ s reported their disappearances verbally. The other half traced their disappearances on an outline "HB" figure. Results showed that the verbal method significantly favored the reporting of elements from the set $H, h, B, b, 1,11,+$. These data suggest that response bias rather than perceptual organization may be responsible for the preponderance of meaningful disappearances noted in previous research.

It is well known that in normal vision continual voluntary and involuntary movements of the eye provide a constantly fluctuating image on the retina. When optical devices (such as a contact lens and mirror system) are used to stop these fluctuations, the viewer typically reports the disappearance or fragmentation of objects in his visual field. Although these techniques were initially devised to study the importance of eye movements in the maintenance of normal vision, Hebb and his colleagues have put them to a somewhat different use, that is to evaluate the relative stability of different parts of a complex figure and to make inferences about underlying perceptual processes. Pritchard, Heron, and Hebb (1960) studied the fragmentation of a variety of visual figures, among them a $4 \mathrm{~B}$ pattern in which the vertical line of the " 4 " and the " $\mathrm{B}$ " were congruent. The Ss' verbal descriptions of the fragmentations were recorded on tape; later analysis of these records led the investigators to conclude that fragmentation was not random; when any part of the " $4 \mathbf{B}$ " figure remained, it almost always contained one or more of the meaningful, complete symbols " 4, " "B," or "3." Later Pritchard (1961) reported comparable results using a slightly modified pattern resembling an "HB" in which the rightmost vertical line of the $H$ was congruent with the vertical of the $\mathbf{B}$.

It has been recognized for many years that disappearances can also be induced by having Ss fixate a visual object while voluntarily restraining head, eye, and blinking movements.Clarke $(1957,1960,1961)$ has suggested (with supporting evidence) that disappearances reported under conditions of voluntary fixation are manifestations of the same process that underlies those obtained with optical stabilization. Additional support for this view was reported by McKinney (1963) who reproduced many of Pritchard's figures in luminous paint on a black background and had his Ss voluntarily restrain head and eye movements while viewing these stimuli in a darkened room. The verbal reports of his observers revealed preponderant "meaningful" fragmentations comparable to those obtained earlier by Pritchard.

Unfortunately these data are equivocal Both voluntary and optical stabilization of the retinal image often produce rapidly changing images and the S's report may have been seriously influenced by those verbal labels that were readily available. Considerable evidence suggests that the $S$ would be hindered in both reporting and remembering those complex fragmentations which were difficult to describe with words. In a previous study, Schuck, Brock, and Becker (1964) demonstrated the effects of response bias on reporting the fragmentations of simple L-shaped or U-shaped luminous figures. Only two kinds of fragmentation were of interest in this study, whether the part that disappeared was a complete line segment or whether it was only a portion of a line. The results showed that assigning simple verbal labels to the line segments in the pattern almost doubled the proportion of line disappearances that were reported. Much of the evidence concerning the effects of verbal labels on the memory for visual form has been recently reviewed by Riley (1962).

The purpose of this study was to compare a verbal and nonverbal method of reporting the fragmentation of a luminous "HB" figure under conditions of voluntary fixation. We predicted that the verbal method would significantly favor a specific set of fragmentations corresponding to common linguistic symbols.

\section{METHOD}

\section{Subjects}

Ss were 58 male and 10 female students who received extra credit in a course in introductory psychology for volunteering. All participants had at least 20-33 monocular vision as measured by the Bausch and Lomb Orthorater.

\section{Apparafus}

$S$ sat in a metal chair in front of a small laboratory table in a light-proof room. The luminous figure was centered at approximately eye level on a flat-black plywood partition just $42 \mathrm{in}$. in front of $\mathrm{S}$. The figure was obtained by mounting a 12-in. square electroluminescent panel (Sylvania PM-144) on the partition, then overlaying the panel with a negative of the HB photographically reproduced on high contrast film. Like the figure used by previous workers, the vertical line of the $B$ was congruent with the right vertical of the $\mathrm{H}$. Overall, the HB was 7 in. high by 8 in. wide and was constructed of lines $1 / 2$ in. wide. Input voltage to the panel was set at 
30 volts so that the luminance was approximately .1 ft-L. When viewed in the darkened room, the pattern appeared as a luminous greenish-grey HB on a dark ground. The table top in front of $\mathrm{S}$ was covered with a black cloth to prevent any reflection.

The room was lighted during intertrial intervals by four 150 watt floodlights mounted behind the plywood partition so that light reflected from the white ceiling provided an illumination at S's head position of about 28 ft-c. Solid-state programming and control modules were used to automatically program the stimulus presentations.

\section{Procedure}

The basic procedure was to obtain many reports of disappearances from each $\mathbf{S}$ during each trial but to use only the last one for later analysis. Random intervals of between 5 and $45 \mathrm{sec}$. were predetermined for each trial and as the first disappearanced following the expiration of the interval was reported, the room lights were turned on ending the trial. This time-sampling technique was used to obtain a representative sample of the kinds of fixation occurring over a short time period, rather than just the first or the last.

Ss in the Verbal Group ( 3 females and 31 males) reported their disappearances verbally throughout, including the disappearance that terminated the trial. These reports were recorded on electromagnetic tape for later aralysis.

Ss in the Tracing Group ( 7 females and 27 males) were provided with sheets of data paper upon which the "HB" figure was printed in faint, dashed lines. In order to lessen any possible verbal influence on the reports of this group, $\mathrm{S}$ indicated fragmentations during the trial interval by pressing a micro-switch and was instructed that one of these reports would be accompanied by the onset of the room lights. When this occurred, he traced on the data paper those portions of the figure that had remained intact, omitting the parts that had disappeared. Aside from these differences regarding the mode of reporting, the instructions were the same for both groups and inciuded the following:

"Notice the pattern that is mounted on the partition in front of you. Under certain conditions part of the pattern will drop out leaving other parts intact. This fragmentation of the pattern is the effect we are studying. For this effect to occur, the pattern must be viewed in the dark. You must fixate on one point only, that is the intersection of these two lines ( $E$ indicates the intersection of the horizontal and rightmost vertical line of the HB figure). Please do not move your eyes from this intersection at any time during a trial. You must keep your head, as well as your eyes, motionless. It is important that you even keep from blinking as long as possible. When fragmentation occurs, describe (trace) the part that remains intact. Make your report (tracing) as accurate and as complete as possible."

In order to avoid the problem of scaling the meaningfulness of each reported fragmentation, we decided to consider only reports of an intact $\mathrm{H}, \mathrm{h}, \mathrm{B}, \mathrm{b}, 1,11$, or + .
Pilot data indicated that these fragmentations would include a large proportion of disappearances that were unambiguous and yet satisfied Pritchard, Heron, and Hebb's (1960) implicit definition of meaningfulness (letters, numerals, common symbols). For the Verbal Group, either the numerals "1" and "11" or the verbal description of one or two vertical lines were accepted for the " 1 " and " 11 " categories. Either "plus" or "cross" were accepted for the "+" category. For the Tracing Group, any crossing pair of vertical and horizontal lines were accepted as a "+." For convenience we shall use the term "meaningful" to refer to a reported fragmentation from this arbitrary set.

Monocular vision was obtained by glasses frames that occluded one eye. All Ss were given six practice trials followed by six experimental trials.

\section{RESULTS AND DISCUSSION}

In the Verbal Group, 29 of the 34 Ss reported at least one meaningful disappearance, as compared to only 12 of the $34 \mathrm{Ss}$ in the Tracing Group. When these frequencies were entered into a 2 by 2 contingency table, the resulting Chi Square was $17.74(p<.001)$.

Thus the results show that verbal and tracing methods of reporting disappearances are not comparable, since the verbal method significantly favored the reporting of elements from a set of common linguistic symbols $(H$, $h, B, b, I, I I,+)$. In conjunction with previous evidence (Schuck, Brock \& Becker, 1964) the present data strongly suggest that response bias rather than perceptual organization may be responsible for the preponderance of meaningful disappearances previously noted by Pritchard, Heron, and Hebb (1960) and McKinney (1963). Moreover, Hebb's recent theoretical statement about perceptual organization (Hebb, 1963), based as it was upon Pritchard's data, must also remain in doubt.

\section{References}

Clarke, F, J, J. Rapid adaptation of localized areas of the extrafoveal retina. Optica Acta, 1957, 4, 69-77.

Clarke, F.J.J. A study of Troxler's effect. Optica Acta, 1860. 7. 219-236.

Clarke, F, J.J. Visual tecovery following local adaptation of the peripheral retina. Optica Acta, 1961, 8, 121-135.

Hebb, D. $\boldsymbol{O}$. The semiautonomous process: its nature and nurture. Amer. Psychologist, 1963, 18, 16-27.

Mckinney, J. P. Disappearance of luminous designs. Science. 1963, 140, 403-405.

Pritchard, R. M. Stabilized images on the retina. Scient. American, $1961,204(6), 72-78$.

Pritchard, R. M, Heron, W., \& Hebb, D. O. Visual perception approached by the method of stabilized images. Canad. 7 . Psychol, $1960,14,67-77$.

Riley, D. A. Memory for form. In L. Postman (Ed.), Psychology in the making. New York: Knopf, 1962. Pp. 402-465.

Schuck, J. R., Brock, T. C., \& Becker, L. A. Luminous figures: factors affecting the reporting of disappearances. Science, 1964 , 146, 1598-1599.

\section{Note}

1. This research was supported by National Institutes of Health Grant MH 10754-01.

(Received in the Editorial Office April 18, 1966.) 